\title{
HALL'S RAY IN INHOMOGENEOUS DIOPHANTINE APPROXIMATION
}

\author{
T. W. CUSICK, W. MORAN and A. D. POLLINGTON
}

(Received 19 August 1993; revised 27 November 1993)

Communicated by J. H. Loxton

\begin{abstract}
The aim of the paper is to show the existence of a 'Hall's ray' for the particular case of the one-sided inhomogeneous diophantine approximation problem, where the irrational is the golden ratio. The proof uses a sum-set method similar to that used by Marshall Hall for the original result of this kind.
\end{abstract}

1991 Mathematics subject classification (Amer. Math. Soc.): 11J06, 11J20.

\section{Introduction}

For a real number $\xi$, the homogeneous approximation constant $\lambda(\xi)$ is defined by

$$
\lambda(\xi)^{-1}=\liminf _{q \rightarrow \infty} q\|q \xi\|,
$$

where $q$ is a positive integer and $\|x\|$ denotes the distance from $x$ to the nearest integer. The set of values taken by $\lambda(\xi)$ as $\xi$ runs through the real numbers is called the Lagrange spectrum. Much is known about the distribution of the numbers in this spectrum (see Cusick and Flahive [3]). In particular, it follows from a result of Hall [6] that there exists a number $w$ such that all numbers in the interval $[w, \infty)$ belong to the Lagrange spectrum (see [3, Chapter 4]). This infinite interval contained in the Lagrange spectrum is called Hall's ray.

The purpose of this paper is to prove that there is an infinite interval, analogous to Hall's ray, contained in the set of inhomogeneous diophantine approximation constants associated with the quadratic irrational $\alpha$ defined by

$$
\alpha=\frac{1}{2}(1+\sqrt{5})
$$

(C) 1996 Australian Mathematical Society 0263-6115/95 \$A2.00+0.00 
Given any real numbers $w, v$ we define the (one-sided) inhomogeneous diophantine approximation constant $k(w, v)$ by

$$
k(\dot{w}, v)^{-1}=\liminf _{q \rightarrow \infty} q\|q w-v\|,
$$

where $q$ is a positive integer. There is also a two-sided inhomogeneous approximation constant $\tau(w, v)$ defined by

$$
\tau(w, v)^{-1}=\liminf _{|q| \rightarrow \infty}|q|\|q w-v\|,
$$

where $q$ is an arbitrary integer. The theory of these two-sided constants is more complicated for the problem we are studying in this paper, so we shall confine our work below to the one-sided constants.

Not much is known about the distribution of the values of the inhomogeneous constants $k(w, v)$, though Barnes [1] gives a proof that for any sufficiently small $r$ there exist uncountably many $w$ and, for each such $w$, uncountably many $v$ such that $k(w, v)=r$. In fact, as Barnes has pointed out to us, the method of proof shows more, namely that for any $w$ whose partial quotients tend to infinity there exist uncountably many such $v$. Thus Barnes produces a Hall's ray for numbers $w$ with partial quotients tending to infinity. His result treats numbers which are at the opposite extreme from the golden ratio in their diophantine approximation properties .

One of the reasons for the paucity of results is the lack of a sufficiently simple analogue of the well-known continued fraction algorithm. It is the use of continued fractions which leads to so many of the results in the homogeneous case. For example, the fact that the lim inf in (1) can be computed by considering only those integers $q$ which occur as denominators of the convergents $p_{n} / q_{n}$ to the continued fraction for $\xi$ greatly facilitates the study of the constants $\lambda(\xi)$. There is a kind of inhomogeneous analogue for the continued fraction which was first given by Cassels [2] and which we shall to some extent utilise below. However, the Cassels algorithm is much more complicated than the continued fraction; in particular, the sequence of integers for which the lim inf in (3) is approached cannot in general be described in a straightforward way. It turns out that when $w=\alpha$ (as defined in (2)), the relevant sequence of integers is relatively simple and can be handled in a satisfactory way by making use of a method of Cusick, Rockett and Szusz [4]. This leads to a proof of the following theorem, which is the main result of this paper.

THEOREM. Given $\alpha=(1+\sqrt{5}) / 2$, there is a number $\Delta$ such that for any number $\gamma$ in the interval $[\Delta, \infty)$, there exists a number $\beta$ for which $k(\alpha, \beta)=\gamma$. An allowable value for $\Delta$ is $\alpha^{3}$. 


\section{An inhomogeneous diophantine approximation algorithm}

This section is based largely on the paper of Cusick, Rockett and Szusz [4]; we shall quote the needed results from that paper without reproducing their proofs.

We use the usual notation

$$
\xi=\left[a_{0}, a_{1}, a_{2}, \ldots\right]
$$

for the regular continued fraction for $\xi$; thus $a_{0}=[\xi]$ ([-] denotes the greatest integer function) and the other partial quotients $a_{k}(k \geq 1)$ are positive integers. We write the $k$-th complete quotient $\xi_{k}$ as

$$
\xi_{k}=\left[a_{k}, a_{k+1}, \ldots\right]
$$

and for the $k$-th convergent $p_{k} / q_{k}$ we have

$$
\frac{p_{k}}{q_{k}}=\left[a_{0}, a_{1}, a_{2}, \ldots, a_{k}\right] .
$$

Finally, we define

$$
D_{k}=q_{k} \xi-p_{k}=(-1)^{k}\left(\xi_{k+1} q_{k}+q_{k-1}\right)^{-1} \quad(k=0,1,2, \ldots) .
$$

We note that

$$
D_{k+1}=a_{k+1} D_{k}+D_{k-1} \quad(k=0,1,2, \ldots)
$$

where $D_{-1}=-1$, and

$$
\frac{D_{k+1}}{D_{k}}=-\xi_{k+2}^{-1} \quad(k=0,1,2, \ldots) .
$$

In order to describe our homogeneous approximation algorithm, we need the following lemma, which is [4, Lemma 1]; an equivalent lemma was given in a paper of Sós [7].

LEMMA 1. Given $\xi=\left[a_{0}, a_{1}, a_{2}, \ldots\right]$ and any real number $\beta$ satisfying $-\xi_{1}^{-1} \leq$ $\beta<1-\xi_{1}^{-1}$, there is a representation of $\beta$ in the form

$$
\beta=\sum_{k=0}^{\infty} c_{k+1} D_{k}
$$

where $c_{k}$ is an integer, $0 \leq c_{1}<a_{1}, 0 \leq c_{k+1} \leq a_{k+1}$ for $k>0$ and $c_{k+1}=a_{k+1}$ implies $c_{k}=0$. The representation is unique apart from changes that could be made using the identities

$$
\sum_{k=0}^{\infty} a_{2 k+n+1} D_{2 k+n}=-D_{n-1} \quad(n=0,1,2, \ldots) .
$$


We shall call an expansion of the form (5) with coefficients $c_{k+1}$ satisfying all of the conditions of Lemma 1 except possibly the condition $0 \leq c_{1}<a_{1}$, a $\xi$-expansion for $\beta$. We do not require $0 \leq c_{1}<a_{1}$ so as to allow $\xi$-expansions for numbers outside of the interval $\left[-\xi_{1}^{-1}, 1-\xi_{1}^{-1}\right)$.

For each pair $w, v$, the inhomogeneous diophantine approximation algorithm of Cassels [2] gives a sequence $S(w, v)$ of integers such that the lim inf in (3) can be computed by considering only those integers $q$ which occur in the sequence $S(w, v)$. Unfortunately, the sequence $S(w, v)$ is in general a complicated one. The elements of $S(w, v)$ need not be distinct and the sequence need not be monotone increasing. Descombes [5, pp. 287-303] gives a detailed exposition of the Cassels algorithm which clearly brings out the erratic nature of the sequence $S(w, v)$. In the case $w=\alpha$ (where $\alpha=(1+\sqrt{5}) / 2$ ), however, we can find a simple set of integers $q$ which will give the lim inf in (3).

LemMA 2. Suppose $\alpha=(1+\sqrt{5}) / 2, \beta$ satisfies $-\alpha^{-1} \leq \beta<1-\alpha^{-1}$ and the $\alpha$-expansion of $\beta$ is given by (5). Then

$$
k(\alpha, \beta)^{-1}=\liminf _{n \rightarrow \infty} \min \left(Q_{n}\left\|Q_{n} \alpha-\beta\right\|,\left(Q_{n}+q_{n}\right)\left\|\left(Q_{n}+q_{n}\right) \alpha-\beta\right\|\right)
$$

where

$$
Q_{n}=\sum_{k=0}^{n} c_{k+1} q_{k} \quad(n=0,1,2, \ldots)
$$

and $q_{k}(k=0,1,2, \ldots)$ is the denominator of the $k$-th convergent $p_{k} / q_{k}$ to $\alpha$.

PROOF. This is part of Lemma 2 of [4].

Both expressions in the minimum in (6) are easy to describe asymptotically. If $D_{k}=q_{k} \alpha-p_{k}$, then it follows from (4) that

$$
D_{k+1} / D_{k}=-\alpha^{-1} \quad(k=0,1,2, \ldots)
$$

so

$$
D_{k}=(-1)^{k}(\alpha-1) \alpha^{-k} \quad(k=0,1,2, \ldots) .
$$

This and the elementary fact that

$$
q_{k}=\alpha^{k+1}-\alpha^{-(k+1)} \sim \alpha^{k+1}
$$

give the asymptotic formula

$$
Q_{n}\left\|Q_{n} \alpha-\beta\right\| \sim \sum_{k=0}^{n} c_{k+1} \alpha^{k}\left|\sum_{k=n+1}^{\infty}(-1)^{k} c_{k+1} \alpha^{-k}\right|,
$$

by using the $\alpha$-expansion (5) of $\beta$. A similar calculation yields a corresponding formula for the other term but as the next lemma shows it is not needed. 
LEMMA 3. For $n$ sufficiently large, the minimum in (6) always occurs at the first of the two terms; that is

$$
\left(Q_{n}+q_{n}\right)\left\|\left(Q_{n}+q_{n}\right) \alpha-\beta\right\| \geq Q_{n}\left\|Q_{n} \alpha-\beta\right\| .
$$

PROOF. Evidently the term $\left(Q_{n}+q_{n}\right)$ is greater than $Q_{n}$. Now consider $\|\left(Q_{n}+\right.$ $\left.q_{n}\right) \alpha-\beta \|$. By Lemma 1,

$$
\beta=\sum_{k=0}^{\infty} c_{k+1} D_{k}, \quad \text { where } c_{k}=0 \text { or } 1
$$

and $D_{k}=q_{k} \alpha-p_{k}$. Therefore

$$
\begin{aligned}
\left\|\left(Q_{n}+q_{n}\right) \alpha-\beta\right\| & =\left\|\sum_{k=0}^{n} c_{k+1} D_{k}+D_{n}-\sum_{k=0}^{\infty} D_{k} c_{k+1}\right\| \\
& =\left\|-\sum_{k=n+1}^{\infty} c_{k+1} D_{k}+D_{n}\right\| \\
& =\left\|\alpha^{-n}(\alpha-1)(-1)^{n}-\sum_{k=n+1}^{\infty} c_{k+1}(-1)^{k} \alpha^{-k}(\alpha-1)\right\|,
\end{aligned}
$$

and for large $n$ this equals

$$
(\alpha-1) \alpha^{-n}\left|1+\sum_{k=1}^{\infty}(-1)^{k-1} c_{k+n+1} \alpha^{-k}\right| .
$$

Let

$$
x_{n}=\sum_{k=1}^{\infty}(-1)^{k-1} c_{k+n+1} \alpha^{-k}=(\alpha-1)^{-1} \alpha^{n}\left\|Q_{n} \alpha-\beta\right\| .
$$

Then for $x_{n} \geq-1 / 2,\left|1+x_{n}\right| \geq\left|x_{n}\right|$ and there is nothing more to prove. If, on the other hand, $x_{n}<-1 / 2$ then $Q_{n} \leq q_{n}+q_{n+2}+q_{n+4}+\cdots$, so that

$$
\frac{Q_{n}+q_{n}}{Q_{n}} \geq 1+\frac{1}{\alpha}
$$

and this is the maximum possible value of $\left|x_{n}\right| /\left|1+x_{n}\right|$. This completes the proof of the lemma.

\section{Proof of Theorem}

Our methods are reminiscent of those of Hall in [6]. We take the logarithm of the first term in the minimum in (6) and then realise the problem as one of showing that 
the sum of two Cantor sets contains an interval. We begin by exploring two Cantor dissections of intervals in $\mathbf{R}$. Let

$$
F=\left\{\sum c_{k}(-1)^{k} \alpha^{-k}: c_{k}, c_{k+1} \text { not both } 1\right\}
$$

and

$$
E=\left\{\sum c_{k} \alpha^{-k}: c_{k}, c_{k+1} \text { not both } 1\right\} .
$$

In fact these two sets are much simpler than they seem.

LEMMA 4. $E=[0,1]$ and $F=\left[-1, \alpha^{-1}\right]$.

PROOF. We will employ the notation $c_{1} c_{2} c_{3} \ldots$ for the sum $\sum_{k=1}^{\infty} c_{k} \alpha^{-k}$ when discussing $E$ and for the sum $\sum_{k=1}^{\infty} c_{k}(-1)^{k} \alpha^{-k}$ when $F$ is under consideration.

We first consider $E$. Evidently the smallest member of $E$ is 0 and the largest value is $1010 \ldots$ which is 1 . Now let $x$ be any element of the interval $(0,1)$. We may write $x$ as a decimal in base $\alpha$. Suppose that this contains a word of the form 11, and let the first occurrence of this 11 be in a situation of the form $w 11 v$. It follows that $w$ has length $k-1$, contains no 11 's, and ends with a 0 . Since $011=100$ we may replace the 011 in the $k-1, k, k+1$ slot by 100 . This may result in a 11 in the new $w$ but in this case it is preceded by a 0 and again the 011 may be replaced by a 100 . Continuing in this way, we can eliminate all 11's from the new $w$ 's as they arise. Note that we cannot finish this process with a $w$ starting with 11 as this number would be not less than 1. Thus we have replaced the expression for $x$ by one with no 11's before the $k+1$ slot. Now we repeat this procedure where necessary to eliminate all 11 's from $x$. This proves the first claim.

Now we look at the set $F$. It is clear that its maximum value occurs at

$$
010101 \ldots=\alpha^{-1}
$$

and its minimum is at $101010 \ldots=-1$. We consider the Cantor dissection process which gives rise to $F$. The largest value in $F$ which starts with the digit 1 is

$$
100101010 \ldots=-\alpha^{-1}+\alpha^{-3}=-\alpha^{-2} .
$$

On the other hand the smallest member of $F$ starting with 0 is

$$
001010101 \ldots=-\alpha^{-2} \text {. }
$$

Hence the 'gap' at the first level of the Cantor dissection has length zero. In general there are two possible kinds of dissections. In the first a word $w$ will have length $k$ odd and end with a 0 and the 'gap' will be between the largest element starting with $w 0$ and the smallest element starting with a $w 1$. The former is $w 001010101 \ldots$ 
and the latter $w 10010101 \ldots$ After subtracting $w$ and rescaling, we have to compare $00101010 \ldots$ with $10101 \ldots$ The former is $-\alpha^{-2}$ and the latter is $-\alpha^{-1}+\alpha^{-3}$. These are identical as in the first level case.

In the second kind of dissection $w$ is a word of length $k$ even ending with a 0 , and we wish to compare the largest word starting with $w 1$ with the smallest word starting with $w 0$. The former is $w 1001010101 \ldots$ and the latter is $w 001010101 \ldots$. Thus after rescaling we compare again $-\alpha^{-1}+\alpha^{-3}$ with $-\alpha^{-2}$ and deduce that there is no gap.

It will be necessary however to replace $E$ and $F$ by two less simple sets. For $k$ a positive integer let $E_{k}$ and $F_{k}$ be the subsets of $E$ and $F$ which are defined by the constraint that the sequence $\left(c_{k}\right)$ contains no block of $k+1$ consecutive zeroes. These are genuine Cantor sets. Nevertheless the product of them contains an interval.

LEMMA 5. For $k \geq 4, E_{k} \cdot\left|F_{k}\right| \supset\left[\alpha^{-2 k}, 1\right]$.

PROOF. We apply Hall's criterion [6] (for deciding whether the sum of two Cantor sets is an interval) to the logarithms of the sets $E_{k}$ and $\left|F_{k}\right|$. This criterion is that the gaps formed at each stage of the Cantor construction are shorter than the adjacent intervals, and that the ratio of the lengths of the initial undissected intervals is between $1 / 3$ and 3 . Evidently $\log E_{k}$ and $\log \left|F_{k}\right|$ satisfy the latter condition.

First we consider $E_{k}$. The smallest point of $E_{k}$ is $\left(0^{k} 1\right)^{\infty}$ and its largest point is the same as that of $E$, namely 1 . However now there is a gap between $(01)^{\infty}$ and the next point in the set, which is $1\left(0^{k} 1\right)^{\infty}$. This is, in fact, the smallest point in $E_{k}$ starting with a 1 . Thus the gap in this case is $\alpha^{-1} /\left(\alpha^{k+1}-1\right)$ whereas the length of the right hand interval is

$$
\alpha^{-2}-\frac{\alpha^{-k-2}}{\left(\alpha^{k+1}-1\right)}
$$

and the left hand interval has length

$$
\alpha^{-1}-\frac{1}{\left(\alpha^{k+1}-1\right)}
$$

The effect of taking logarithms can be estimated in terms of the derivative of log. This has the effect of expanding the left hand interval relative to the gap and so merely improves the ratio between the length of the left hand interval and the gap. At the . right hand end the effect of the logarithm is to shrink the interval by an amount of no more than $\alpha$ relative to the gap, since the lower endpoint of the gap is at $\alpha^{-1}$ and the upper endpoint of the interval is at 1 . Thus the criterion is satisfied at the first level for $E_{k}$ provided $k \geq 2$.

At the next level the four intervals in the Cantor dissection for $E_{k}$ are

$$
\left[\left(0^{k} 1\right)^{\infty}, 0(01)^{\infty}\right], \quad\left[01\left(0^{k} 1\right)^{\infty},(01)^{\infty}\right], \quad\left[1\left(0^{k} 1\right)^{\infty}, 1(01)^{\infty}\right], \quad\left[11\left(0^{k} 1\right)^{\infty}, 1\right] .
$$


Essentially the same calculation as we have already done at the first level shows that the gaps are shorter than the adjacent intervals. Indeed the first two intervals are merely the intervals of the first level scaled down by a factor of $\alpha^{-1}$ and the second two are translations of the first two intervals by $\alpha^{-1}$. The effect of taking logarithms on the first two intervals is the same as at the first level, and the effect on the second two intervals is less severe since they are further away from 0 . Thus the criterion is again satisfied at this level. It is now clear that the argument will continue to work at all succeeding levels provided $k \geq 2$.

In the case of $F_{k}\left(k\right.$ even) it is convenient to restrict attention to $\left(-F_{k}\right) \cap[0,1]$. For this the smallest point is $0^{k} 1001(01)^{\infty}$ and the largest point is again 1 . The first level gap has left hand endpoint $10^{k} 1001(01)^{\infty}$ and right hand endpoint $10^{k-1} 10(01)^{\infty}$. Subsequent dissections are obtained by scaling and translating this one. The lengths of the left and right intervals and gap in the initial dissection are respectively

$$
\begin{aligned}
& \alpha^{-1}-\alpha^{-(k+2)}+\frac{\alpha^{-(k+5)}}{\left(1-\alpha^{-2}\right)}-\alpha^{-(k+1)}+\frac{\alpha^{-(k+4)}}{\left(1-\alpha^{-2}\right)}, \\
& 1-\alpha^{-1}-\alpha^{-(k+1)}+\frac{\alpha^{-(k+4)}}{\left(1-\alpha^{-2}\right)} \\
& \alpha^{-(k+1)}+\alpha^{-(k+2)}-\frac{\left(\alpha^{-(k+4)}+\alpha^{-(k+5)}\right)}{\left(1-\alpha^{-2}\right)} .
\end{aligned}
$$

Again it is easy to check that Hall's criterion is satisfied at this level provided in this case that $k \geq 4$. At subsequent levels, as in the case of $E_{k}$, the situation is no worse.

Since the sets $F_{k}$ are nested $\left(F_{k} \subset F_{k+1}\right)$, the result remains true for $k$ odd.

Now fix $x$ in the interval $I_{1}=\left[\alpha^{-10}, \alpha^{-3}\right]$ and choose members $e$ and $f$ of the sets $E_{4}$ and $-F_{4} \cap[0,1]$ respectively such that $e \cdot f=\alpha^{3} x$. Let $e=\sum_{k=1}^{\infty} e_{k} \alpha^{-k}$ and $f=$ $\sum_{k=1}^{\infty}(-1)^{k+1} f_{k} \alpha^{-k}$. We define $\left(c_{k}\right)$ as follows. Let $c_{1}, c_{2}, c_{3}, c_{4}, c_{5}$ be the first five members of the sequence for $e$ in the reverse order, that is $e_{5}, e_{4}, e_{3}, e_{2}, e_{1}$. The next five members of $\left(c_{k}\right)$ are all 0 , and the following five members are $f_{1}, f_{2}, f_{3}, f_{4}, f_{5}$. Now we repeat the same process with the first 10 members of the sequence for $e$ in reverse order followed by 50 's and then the first ten members of the sequence for $f$. We continue in this way taking in turn a block of length $5 r$ from the beginning of $\left(e_{k}\right)$ in reverse order followed by five 0 's and then a block of length $5 r$ from the beginning of $\left(f_{k}\right)$. In this way the sequence $\left(c_{k}\right)$ is constructed.

Now we use the formula (6), or at least the first term in the minimum. The infimum of the expression

$$
\sum_{k=0}^{n} c_{k+1} \alpha^{k}\left|\sum_{k=n+1}^{\infty}(-1)^{k} c_{k+1} \alpha^{-k}\right|
$$

over $n \geq N$, say, is taken at a point $n$ which lies in a block of 5 zeroes. Thus, by the construction of the sequence, the lim inf is the limit of 


$$
\alpha^{-3}\left(\sum_{k=1}^{5 r} e_{k} \alpha^{-k}+\delta_{r}\right)\left|\sum_{k=1}^{5 r}(-1)^{k} f_{k} \alpha^{-k}+\epsilon_{r}\right| \text {, }
$$

as $r$ tends to infinity, where

$$
\delta_{r}=o\left(\sum_{k=1}^{5 r} e_{k} \alpha^{-k}\right)
$$

and

$$
\epsilon_{r}=o\left(\sum_{k=1}^{5 r}(-1)^{k} f_{k} \alpha^{-k}\right)
$$

This limit is therefore equal to $x$. It follows that the interval $I_{1}$ is contained in the spectrum.

Now we perform the same construction for $\left(c_{k}\right)$ except that we intersperse the blocks of $e_{k}$ 's and $f_{k}$ 's with blocks of 6 zeroes. This reduces the lim inf by a factor of $\alpha^{-1}$. It follows that $I_{2}=\alpha^{-1} I_{1}$ is also in the spectrum. Evidently by increasing the number of zeroes interspersing the terms from the sequences $\left(e_{k}\right)$ and $\left(f_{k}\right)$ we can cover the whole of the interval $\left[0, \alpha^{-3}\right]$. This interval is, therefore, in the spectrum and constitutes a 'Hall's ray' for the inhomogeneous minimum problem.

\section{References}

[1] E. S. Barnes, 'On linear inhomogeneous diophantine approximation', J. London Math. Soc. 31 (1956), 73-79.

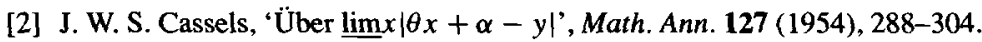

[3] T. W. Cusick and M. E. Flahive, The Markoff and Lagrange spectra, Mathematical Surveys and Monographs 30 (Amer. Math. Soc., Providence, 1989).

[4] T. W. Cusick, A. M. Rockett and P. Szüsz, 'On inhomogeneous diophantine approximation', J. Number Theory 48 (1994), 259-283.

[5] R. Descombes, 'Sur la repartition des sommets d'une ligne polygonale reguliere non fermée', Ann. Sci. Ecole Norm. Sup. (3) 73 (1956), 283-355.

[6] M. Hall, Jr., 'On the sum and product of continued fractions', Ann. of Math. (2) 48 (1947), 966-993.

[7] V. Sós, 'On the theory of diophantine approximations, II', Acta Math. Hungar. 9 (1958), 229-241.

Department of Mathematics

SUNY at Buffalo

NY 14214-3093

USA

Department of Mathematics

Brigham Young University

Provo, UT 84602

USA
School of Information Science and Technology Flinders University GPO 2100, SA5001

Australia : 